\title{
NEW SIGNAL-SPACE ORTHONORMAL BASES VIA THE METAPLECTIC TRANSFORM
}

\author{
Richard G. Baraniuk ${ }^{1}$ and Douglas L. Jones ${ }^{2}$ * \\ ${ }^{1}$ Laboratoire de Traitement du Signal, Laboratoire de Physique \\ Ecole Normale Supérieure de Lyon \\ 46 allée d'Italie, 69364 Lyon Cedex 07 France \\ ${ }^{2}$ Department of Electrical and Computer Engineering \\ University of Illinois, Urbana, IL 61801
}

\begin{abstract}
The two primary classes of time-frequency-concentrated orthonormal bases (ONBs) can be in terpreted as arising from the discretization of either the short-time Fourier transform (STFT) or the continuous wavelet transform (CWT). Recently, the five-dimensional metaplectic transform (MT) has been proposed as a generalization of the STFT and CWT. It allows shears and rotations of the analyzing window/wavelet in the time-frequency plane, in addition to translations and scaling. Just as the CWT and STFT can be discretized on lattices of points in two dimensions, the MT can be discretized on lattices of points in five dimensions. In this paper, we consider the discretization of the MT, and show that it can lead to entirely new ONBs for the signal space of square integrable functions. Two new classes of bases, the scale and shear bases and the translation and shear bases, are derived to demonstrate the discretization process. Besides generalizing the current methods of generating time-frequency-concentrated $\mathrm{ONBs}$, MT bases possess extra degrees of freedom that can be used to match a wider variety of signals.
\end{abstract}

\section{INTRODUCTION}

The short-time Fourier transform (STFT)

$$
\zeta_{s}(t, \omega)=\int s(\tau) g_{\mathrm{stft}}^{*}(\tau-t) e^{-j \omega \tau} d \tau
$$

and the continuous wavelet transform (CWT)

$$
\psi_{s}(t, a)=|a|^{-\frac{1}{2}} \int s(\tau) g_{\mathrm{cwt}}^{*}\left(\frac{\tau-t}{a}\right) d \tau
$$

are time-frequency representations that map a onedimensional signal $s$ to the two-dimensional time-frequency and time-scale planes [1]. Both have been utilized in many signal processing applications to analyze the time-varying frequency content of nonstationary signals. The function $g$ is known as the window in the STFT and as the wavelet in the CWT.

While the STFT and CWT are important time-frequency analysis tools in their own right, attention has focused recently on using these transforms (especially the CWT) to

*This work was supported by the Sound Group of the Computer-Based Education Research Laboratory at the University of Dllinois, the Joint Services Electronics Program, Grant No. No0014-90-J-1270, and the National Science Foundation, Grant No. MIP 90-12747. R. Baraniuk is on leave from Rice University, P.O. Box 1892, Houston, Texas 77251-1892, USA. construct orthonormal bases ${ }^{1}$ (ONBs) for the signal space of square integrable functions $L^{2}(\mathbb{R})[1]$. Obtained by discretizing the STFT or CWT on a lattice of points in the time-frequency plane, these ONBs have the useful property that the basis elements are local in both time and frequency, unlike the well-known Fourier basis elements, which are local in frequency but global in time.

STFT basis expansions are constructed by discretizing the time and frequency translates of the window function utilized in (1) on a uniform lattice of points

$$
t=m t_{0}, \quad \omega=n \omega_{0}, \quad m, n \in Z
$$

in the time-frequency plane. For certain choices of $\left(g_{s t f t}, t_{0}, \omega_{0}\right)$, these functions form an ONB for $L^{2}(\mathbb{R})$, and we have the following signal expansion [1]:

$$
s(\tau)=\sum_{m} \sum_{n} c_{m, n}^{\mathrm{stft}} b_{m, n}^{\mathrm{stft}}(\tau), \quad s \in L^{2}(\mathbb{R})
$$

Basis elements: $b_{m, n}^{\mathrm{stft}}(\tau)=g_{\mathrm{sttt}}\left(\tau-m t_{0}\right) e^{j n \omega_{0} r}$

Coefficients : $\quad c_{m, n}^{s t f t}=\left\langle s, b_{m, n}^{s t f t}\right\rangle=\zeta_{s}\left(m t_{0}, n \omega_{0}\right)$

The inner product in $L^{2}(\mathbb{R})$ is defined as $\langle g, h\rangle=$ $\int g(u) h^{*}(i) d u$, so the STFT values evaluated on the lattice form the coefficients of the expansion. The basis elements are often called logons, and, as shown in Fig. 1(a), they "tile" the time-frequency plane in a constant-bandwidth fashion. One simple window that leads to an ONB (when $\left.t_{0}=1, \omega_{0}=2 \pi\right)$ is the rectangle function

$$
g_{\text {rect }}(u)= \begin{cases}1, & 0<u \leq 1 \\ 0, & \text { otherwise }\end{cases}
$$

ONBs can also be constructed from the CWT, by discretizing the time-translated and scaled wavelets from (2) on an exponential lattice of points

$$
t=m t_{0} a_{0}^{n}, \quad a=a_{0}^{n}, \quad m, n \in Z
$$

in the time-scale plane. To make the following discussion more transparent, we discuss CWT bases only for analytic signals ${ }^{2}$ in the Hardy space $H^{2}(\mathbb{R})$. Basis construction methods for $L^{2}(\mathbb{R})$ are completely similar to the $H^{2}(\mathbb{R})$ case [1]. For certain choices of $\left(g_{\mathrm{cwt}}, t_{0}, a_{0}\right)$, the discretized wavelet logons form an ONB for $H^{2}(\mathbb{R})$, and the following

\footnotetext{
${ }^{1}$ An orthonormal basis is defined as a set of vectors $\left\{b_{i}\right\}$ in a Hilbert space that is normalized, mutually orthogonal, and complete.

${ }^{2}$ Recall that the Fourier transform $H$ of an analytic signal $h$ is one-sided; that is, $H \in L^{2}\left(\mathbb{R}_{+}\right)$.
} 
signal representation results:

$$
s(\tau)=\sum_{m} \sum_{n} c_{m, n}^{c w t} b_{m, n}^{c w t}(\tau), \quad s \in H^{2}(\mathbb{R})
$$

Basis elements: $b_{m, n}^{c m t}(\tau)=a_{0}^{-n / 2} g\left(a_{0}^{-n} \tau-m t_{0}\right)$

Coefficients : $\quad c_{m, n}^{c+1}=\left\langle s, b_{m, n}^{c+t}\right\rangle=\psi_{*}\left(m t_{0} a_{0}^{n}, a_{0}^{n}\right)$.

The Fourier transform of a CWT basis element is given by

$$
B_{m, n}^{c W t}(\Omega)=a_{0}^{n / 2} G_{c m t}\left(a_{0}^{n} \Omega\right) e^{-j a_{0}^{n} \Omega m t_{0}} .
$$

As we see from Fig. 1(b), the CWT logons "tile" the timefrequency plane in a proportional-bandwidth (constant-Q) fashion. A class of highly localized wavelets that yield ONBs has been derived by Daubechies [2].

The discretized STFT and CWT represent two approaches to tiling the time-frequency plane. Both sets of bases have received considerable attention recently, not only because of their elegant theoretical structure, but also because of their localization properties, which are desirable in a wide variety of important applications. In fact, the derivation of CWT bases with remarkable properties is perhaps the primary cause of the recent surge of interest in the CWT in particular and time-frequency analysis in general.

However, there exist signals that are well modeled by neither a constant-bandwidth (STFT) nor a constant- $Q$ (CWT) style of analysis. Examples include chirping or dispersed signals that can only be matched well by slanted (chirping) logons. Therefore, in this paper, we present a method for generating new ONBs with extra degrees of freedom that allow the basis elements to shear, or chirp, and even rotate in the time-frequency plane, in addition to translate and scale. The general class of ONBs encompasses the bases generated by both the STFT and CWT as special cases. Besides the theoretical benefit of uniting these two types of expansions, the other $\mathrm{ONBs}$ from this new class could possibly produce better results (packing more energy into fewer expansion coefficients, for example) than existing bases for certain types of signals, since the extra degrees of freedom can be used to better match the basis elements to the signal being represented. The new method is based on discretizing the metaplectic transform. This transform was introduced in [3], [4]; we present a brief summary below.

\section{THE CONTINUOUS METAPLECTIC TRANSFORM}

In the STFT and CWT, the transformations applied to the window/wavelet are limited to time-frequency tranglation and time-frequency scaling. The metaplectic transform (MT) is constructed to implement a general $2 \times 2$ unimodular affine transformation, $A x+b,|A|=1$, in timefrequency [4]. The extra degrees of freedom in this transformation allow logons to not only translate and scale in the time-frequency plane, but also to shear and rotate. Figure 2 shows several logons obtained using this more general time-frequency transformation.

Given a generalized wavelet function $g$, the MT of a signal $s$ is computed as

$$
\Gamma_{s}(t, \omega, a, p, q)=\left\langle s, \overline{\mathcal{F}}_{\omega} \bar{T}_{t} \bar{A}_{a} \overline{\mathcal{Q}}_{q} \overline{\mathcal{P}}_{p} g\right\rangle .
$$

The operators with overbars correspond to frequency translation $\left(\overline{\mathcal{F}}_{\omega} g(\tau)=g(\tau) e^{j \omega \tau}\right)$, time translation $\left(\overline{\mathcal{T}}_{t} g(\tau)=\right.$ $g(\tau-t))$, time-scaling $\left(\mathcal{A}_{a} g(\tau)=|a|^{-\frac{1}{2}} g\left(a^{-1} \tau\right)\right)$, shear along the frequency axis $\left(\overline{\mathcal{Q}}_{q} g(\tau)=g(\tau) e^{j \tau^{2} q / 2}\right)$, and shear along the time axis $\left(\overline{\mathcal{P}}_{p} g(\tau)=(-j p)^{-\frac{1}{2}} e^{j r^{2} / 2 p} \star g(\tau)\right.$, with - denoting convolution). Note that the two shear transformations are implemented using linear-FM ("chirp") signals.

The most noticeable feature of the MT is that it is fivedimensional (5-d); that is, it maps a 1-d time signal to a 5-d analysis space. By contrast, both the STFT and CWT are 2-d transformations. All five dimensions of the MT need not be utilized, however. New 2, 3, and 4-d transformations can be constructed by restricting the computation of the MT to subsets of the 5-d space. Examples of 2-d transforms that reside in subsets of the analysis space include the aforementioned STFT and CWT, which lie on the planes parameterized by $(t, \omega, 1,0,0)$ and $(t, 0, a, 0,0)$, the new scale and shear transform, which lies on the plane $(0,0, a, p, 0)$, and the new time and shear transform, which lies on the plane $(t, 0,1,0, q)$. The scale and shear transform transform $\sigma_{d}$ is computed using

$$
\begin{gathered}
\sigma_{s}(a, p)=\Gamma_{\bullet}(0,0, a, p, 0)=\left\langle s, \overline{\mathcal{A}}_{a} \overline{\mathcal{P}}_{p} g_{s s t}\right\rangle= \\
\int s(\tau)\left[(-j p)^{-\frac{1}{2}}|a|^{-1 / 2} g_{s s t}\left(a^{-1} \tau\right) \star e^{j\left(a^{-1} \tau\right)^{2} / 2 p}\right]^{*} d \tau,
\end{gathered}
$$

while the time and shear transform $\beta_{s}$ is computed using

$$
\begin{aligned}
\beta_{s}(a, p) & =\Gamma_{s}(t, 0,1,0, q)=\left\langle s, \bar{T}_{t} \overline{\mathcal{Q}}_{q} g_{t a t}\right\rangle \\
& =\int s(\tau) g_{t s t}^{*}(\tau-t) e^{j(\tau-t)^{2} q / 2} d \tau .
\end{aligned}
$$

\section{DISCRETIZING THE METAPLECTIC} TRANSFORM

Just as the STFT and CWT can be discretized on lattices in the 2-d time-frequency and time-scale planes, the MT can be discretized on lattices in the 5-d analysis space. The lattices can either be distributed throughout the 5-d space, or be restricted to subsets. Furthermore, ONBs can be constructed from the MT on these lattices. So far, we have found new ONBs by discretizing the MT on lattices that lie in the 2-d $(t, q)$ time and shear, $(\omega, p)$ frequency and shear, $(a, p)$ scale and shear, and $(a, q)$ scale and shear planes. A salient feature of all of these new bases is that they utilize chirp functions to represent signals. To illustrate the specific techniques employed, we now discretize the $(a, p)$ scale and shear and $(t, q)$ time and shear transforms.

\subsection{Scale and Shear Bases}

As with the STFT and CWT, a discretized set of logons comprises an $(a, p)$ scale and shear ONB. We obtain the scale and shear basis elements by discretizing $a$ and $p$ in the continuous-variable logons contained in the square bracket in (12). Like the CWT, we work in the space $H^{2}(\mathbb{R})$ and utilize an exponential lattice

$$
p=m p_{0}, \quad a=r_{0}^{n}, \quad m, n \in Z,
$$

which yields our proposed basis

$$
\left\{\overline{\mathcal{A}}_{r_{0}^{n}} \overline{\mathcal{P}}_{\text {mpo }} g_{s a t}(r)\right\}_{(m, n) \in Z^{2}} \text {. }
$$

If and when there exists a triple $\left(g_{\text {sat }}, r_{0}, p_{0}\right)$ such that (15) is an ONB, we have the following signal expansion:

$$
s(\tau)=\sum_{m} \sum_{n} c_{m, n}^{s s t} b_{m, n}^{s s t}(\tau), \quad s \in H^{2}(\mathbb{R})
$$




$$
\begin{aligned}
& \text { Basis elements: } b_{m, n}^{\mathrm{sst}}(\tau)=\overline{\mathcal{A}}_{r_{0}^{n}} \overline{\mathcal{P}}_{m p_{0}} g_{s s t}(\tau)= \\
& \left(-j m p_{0}\right)^{-\frac{1}{2}}\left[r_{0}^{-n / 2} g_{s s t}\left(r_{0}^{-n / 2} \tau\right) \star e^{j\left(r_{0}^{-n / 2} \tau\right)^{2} / 2 m p_{0}}\right] \\
& \text { Coefficients : } \quad c_{m, n}^{s s t}=\left\langle s, b_{m, n}^{s s t}\right\rangle=\sigma_{o}\left(r_{0}^{n}, m p_{0}\right) .
\end{aligned}
$$

Evaluation of the scale and shear transform at the lattice points conveniently forms the coefficients of the expansion. The Fou rier transform of one of the proposed basis functions is given by

$$
\mathcal{F}\left[b_{m, n}^{8 s t}\right](\Omega)=r_{0}^{n / 2} G_{\mathbf{s t}}\left(r_{0}^{n} \Omega\right) e^{-j\left(r_{0}^{n} \Omega\right)^{2} m p_{0} / 2} .
$$

Note - except for the square in the exponential - the similarity to the Fourier transform of the CWT basis elemen in (10). The logon tiling for an idealized scale and shear basis is shown in Fig. 3. The tilt of the logons suggests that scale and shear bases could prove useful for representing chirp signals.

We now establish the validity of the expansion (16), (17). Remarkably, a simple change of variable will yield all generalized wavelets $g_{s s t}$ that generate scale and shear ONBs on the lattice (14). We omit many of the details of the derivation; see [5] for more details.

Define the axis warping operator $\Lambda_{c}: L^{2}\left(\mathbb{R}_{+}\right) \rightarrow L^{2}\left(\mathbb{R}_{+}\right)$ by $\Lambda_{c} h(v)=\sqrt{c v^{c-1}} h\left(v^{c}\right), c \neq 0, v \in \mathbb{R}_{+}$. We will consider only the case $c=2$ in this paper. Form the threecomponent operator $\Theta=\mathcal{F}^{-1} \Lambda_{2} \mathcal{F}$. For us, the key property of $\Theta$ is that it is unitary, mapping $H^{2}(\mathbb{R})$ on to itself in a one-to-one, isometric (angle-preserving) fashion.

Recall that unitary operators map ONBs to ONBs, so if we apply $\Theta$ to a CWT basis for $H^{2}(\mathbb{R})$, the result will be another basis for $H^{2}(\mathbb{R})$. It is straightforward to show that the basis elements resulting from the application of $\Theta$ to an arbitrary CWT basis $\left\{b_{m, n}^{\mathrm{cWt}}\right\}$ take the form of the scale and shear basis elements $\left\{b_{m_{n}}^{a s t}\right\}$ postulated in (17). That is, $\Theta$ maps every CWT basis to a scale and shear basis. Furthermore, this procedure generates all scale and shear bases, because the inverse of the three-component operator, $\Theta^{-1}=\mathcal{F} \Lambda_{2}^{-1} \mathcal{F}^{-1}$, is also unitary, and hence, it maps every scale and shear basis to a CWT one. This fundamental result is summarized in the following theorem.

Theorem 1 The triple $\left(g_{\mathrm{cwt}}, t_{0}, a_{0}\right)$ generates a $C W T$ $O N B$ for $H^{2}(I R)$ if and only if the triple $\left(g_{\mathrm{sst}}, 2 t_{0}, a_{0}^{1 / 2}\right)$ with $g_{\mathrm{sst}}=\Theta g_{\mathrm{cwt}}$, generates a scale and time-shear $O N B$ for $H^{2}(I R)$

Note that the sampling lattices of the two bases are subtly different. Most conspicuous is the $a_{0}^{1 / 2}$ spacing in scale in the scale and shear basis (typically $a_{0}=2$ ).

The computation of the expansion coefficients $\left\{c_{m, n}^{s 8 t}\right\}$ in (17) appears cumbersome, but it can be efficiently implemented by again utilizing the special properties of the operator $\Theta$. The isometry of this operator from $H^{2}(\mathbb{R})$ on to $H^{2}(\mathrm{IR})$ allows us to compute the expansion coefficients by first "pre-warping" the signal and then computing the CWT basis coefficients:

$$
\begin{aligned}
c_{m, n}^{\mathrm{sst}} & =\left\langle s, b_{m, n}^{\mathrm{sst}}\right\rangle=\left\langle\Theta^{-1} s, \Theta^{-1} b_{m, n}^{\mathrm{sst}}\right\rangle \\
& =\left\langle\Theta^{-1} s, b_{m, n}^{\mathrm{cwt}}\right\rangle .
\end{aligned}
$$

Efficient computation of the prewarped signal $\Theta^{-1} s$ should be possible using techniques analogous to the fast Mellin transform, which requires a geometric scaling of the transform axis [6].

\subsection{Time and Shear Bases}

Mathematical machinery similar to that of the previous section can be applied to construct bases from the translation and shear transforms. To avoid extra complications, we will work in the space of one-sided time signals $L^{2}\left(\mathbb{R}_{+}\right)$. Basis construction for all of $L^{2}(\mathbb{R})$ follows by a straightforward extension of these techniques.

The translation and shear basis elements are obtained by discretizing $t$ and $q$ in the continuous-variable logons of (13) on a uniform lattice

$$
t=m t_{0}, \quad q=n q_{0}, \quad m \in Z_{+}, n \in Z .
$$

The resulting proposed basis for $L^{2}\left(\mathbb{R}_{+}\right)$is

$$
\begin{aligned}
& \left\{\overline{\mathcal{T}}_{m t_{0}} \overline{\mathcal{Q}}_{m q_{0}} g_{\mathrm{tst}}(\tau)\right\}_{(m, n) \in Z_{+} \times Z} \\
& \quad=\left\{g_{\mathrm{tst}}\left(\tau-m t_{0}\right) e^{j\left(r-m t_{0}\right)^{2} n q_{0} / 2}\right\}_{(m, n) \in Z_{+} \times Z} .
\end{aligned}
$$

Save for the chirp factor, the basis elements closely resemble STFT basis elements. The logon tiling for an idealized translation and shear basis is shown in Fig. 4.

The similarity between (21) and (18) suggests that application of the axis warping operator $\Lambda_{2}$ to an STFT ONB for $L^{2}\left(\mathbb{R}_{+}\right)$may result in a proper translation and shear ONB. However, the resulting basis $\left\{b_{m, n}^{\text {tst }}\right\}=\left\{\Lambda_{2} b_{m, n}^{\text {stit }}\right\}$ has the drawback of not being generated by translates and shears of the single wavelet $g_{\mathrm{tss}}$. Instead, we use a different change of variable, related to $\Lambda_{2}$, that yields a large class of related ONBs. We take $t_{0}=1$ from this point.

Define the resetting axis warping operator $\Upsilon_{2}: L^{2}\left(\mathbb{R}_{+}\right) \rightarrow$ $L^{2}(\mathbb{R}+)$ by $\Upsilon_{2} h(u)=\sqrt{2(u-\lfloor u\rfloor)} h\left((u-\lfloor u\rfloor)^{2}+\lfloor u\rfloor\right)$, $u \in \mathbb{R}_{+}$. The effect of $\Upsilon_{2}$ is to periodically (between each integer) warp the scale of the function $h$ and multiply it by a periodic $u^{1 / 2}$ window. Figure 5 illustrates the effect of $\Upsilon_{2}$ on several STFT basis elements generated using the rectangular window of $(6)$. Note how $\Upsilon_{2}$ warps the sinusoids in the STFT basis elements to chirp functions.

Like the three-part operator $\Theta$ from Section 3.1, $\Upsilon_{2}$ is also unitary. Therefore, it maps each ONB for $L^{2}\left(\mathbb{R}_{+}\right)$to another ONB for $L^{2}\left(\mathbb{R}_{+}\right)$. Application of $\Upsilon_{2}$ to the STFT basis elements from (5) results in the basis $\left\{b_{m, n}^{\text {reset }}\right\}$, whose elements are given by

$$
b_{m, n}^{\text {reset }}(\tau)=g_{\text {reset }}(\tau-m) e^{j n \omega_{0}\left[(\tau-\lfloor\tau\rfloor)^{c}+\lfloor\tau\rfloor\right]},
$$

with $g_{\text {reset }}(\tau)=\Upsilon_{2} g_{s t i t}(\tau)$.

The basis $\left\{e_{m, n}^{\text {reset }}\right\}$ has the advantage of being generated by the single function $g_{\text {reset }}$. However, note that the chirping shear component "resets" whenever $\tau$ passes an in teger Thus, in general, the elements $b_{m, n}^{\text {reset }}$ do not arise from discretizing the metaplectic transform, and therefore, $\left\{b_{m, n}^{\text {reset }}\right\}$ is not strictly a metaplectic basis. We call such a basis a quasi translation and shear basis. The following theorem summarizes our results.

Theorem 2 The triple $\left(g_{\mathrm{ttft}}, t_{0}, \omega_{0}\right)$ generates an $S T F T$ $O N B$ for $L^{2}\left(\mathbb{R}_{+}\right)$if and only if the triple $\left(g_{\text {reset }}, t_{0}, 2 \omega_{0}\right)$, where $g_{\text {reset }}=\Upsilon_{c} g_{\mathrm{stft}}$, generates a quasi translation and shear $O N B$ for $L^{2}\left(R_{+}\right)$as in (22). 


\section{CONCLUSIONS}

The MT, a five-dimensional generalization of the STFT and CWT, yields new tilings of the time-frequency plane by allowing logons to shear and rotate. Discretization of these tilings leads to entirely new classes of ONBs. An alternate interpretation of the two example bases we have derived is as direct generalizations of the STFT and CWT constructions: we replace the time-shift $t$ of a CWT basis by the time-shear $p$ of a scale and shear basis, and replace the frequency-shift $\omega$ of an STFT basis by the frequency-shear $q$ of a time and shear basis. While theoretically interesting, these new bases conld possibly also be useful for representing certain types of signals, such as chirps, since the extra degrees of freedom in the MT permit a better matching of the transform to a signal. A potential application of this matching concept is an adaptive $O N B$, where not only the wavelet, but also the time-frequency tiling, is adapted to a given signal. Finally, note that other new bases outside of the metaplectic framework can easily be derived by utilizing $c \neq 2$ in the axis warping operators. These bases represent signals in terms of hyper-chirp functions $e^{j \tau^{c}}$.

(a)

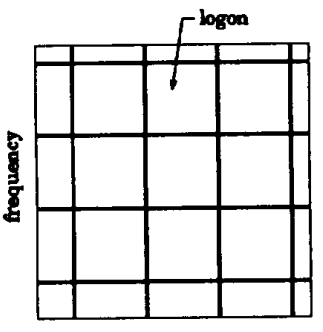

time

\section{REFERENCES}

1. C. Heil and D. Walnut, "Continuous and Discrete Wavelet Transforms," SIAM Review, Vol. 31, No. 4, pp. 628-666, Dec. 1989.

2. I. Daubechies, "Orthonormal Bases of Compactly Supported Wavelets," Comm. on Pure and App. Math., Vol. XLI, pp. 909-996, 1988.

3. S. Mann and S. Haykin, "The Chirplet Transform: A Generalization of Gabor's Logon Transform," Vision Interface91.

4. R. G. Baraniuk and D. L. Jones, "New Dimensions in Wavelet Analysis," ICASSP92, San Francisco, 1992.

5. R. G. Baraniuk and D. L. Jones, "Shear Madness: Discretizing the Metaplectic Transform," preprint.

6. J. Bertrand, et al., "Discrete Mellin Transform for Signal Analysis," ICASSP90, Toronto, 1990.

(b)

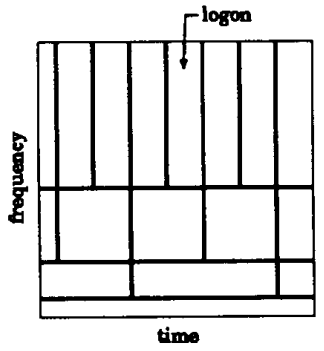

Fig: 1: Time-frequency plane tilings of (a) the STFT and (b) the CWT.

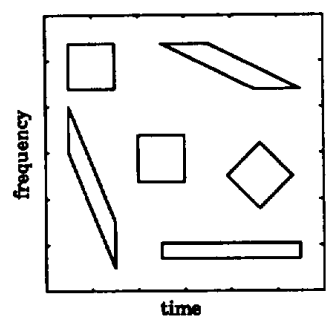

Fig. 2: Logons transformed by 2d unimodular affine transformations.

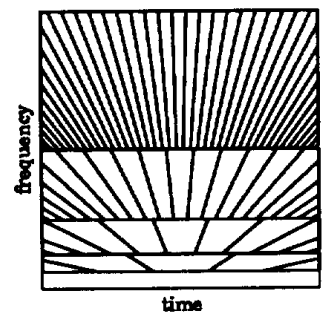

Fig. 3: Scale and shear transform logons tiling the timefrequency plane.

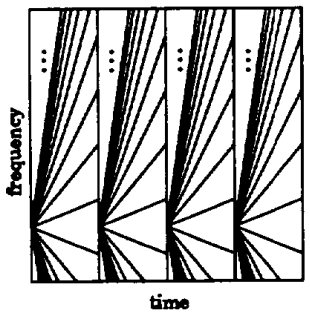

Fig. 4: Time and shear transform logons tiling the timefrequency plane. (a)

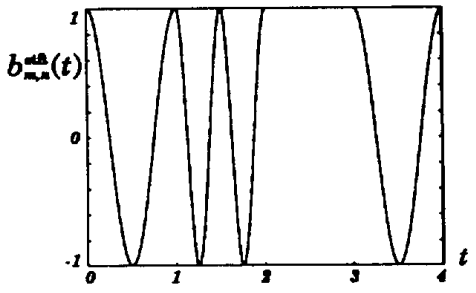

(b)

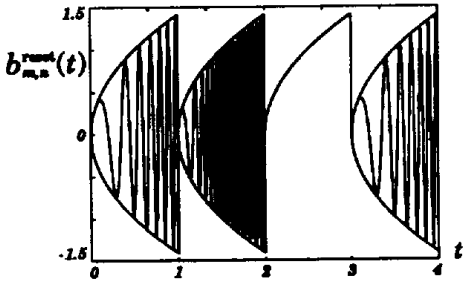

Fig. 5: (a) Several rectangular window basis elements $b_{m, n}^{\text {stft }}(t)$ for $(m, n)=(0,1),(1,2),(2,0),(3,1)$ and $t_{0}=1$, $\omega_{0}=2 \pi$. (b) Quasi translation and shear basis elements $b_{m, n}^{\text {reset }}(t)=\Upsilon_{2} b_{m, n}^{\text {stft }}(t)$. 